\title{
Imagem corporal e autoestima em homens estudantes universitários
}

\section{Body image and self-esteem in male university students}

\begin{tabular}{|c|c|}
\hline $\begin{array}{l}\text { (D) José Mendes }{ }^{1,2} \bowtie \\
\text { (D) Fátima Amaral } \\
\text { (D) Cátia Moniz } \\
\text { (D) Samanta Câmara } \\
\text { (D) Teresa Medeiros } \\
\text { (D,3 }\end{array}$ & $\begin{array}{l}\text { [1] INTELECTO - Psicologia \& Investigação, Ponta Delgada, Açores, Portugal } \\
\text { [2] Instituto de Psicologia Cognitiva, Desenvolvimento Humano e Social, Coimbra, Portugal } \\
\text { [3] Universidade dos Açores, Ponta Delgada, Açores, Portugal } \\
\square \text { josemendes@intelecto.pt }\end{array}$ \\
\hline
\end{tabular}

\section{Resumo}

Objetivo: O presente estudo pretende avaliar diferenças e relações entre o grau de insatisfação com a imagem corporal, o índice de massa corporal (IMC), a prática de exercício físico, a autoestima, a média académica e a satisfação com a vida académica, numa amostra de estudantes do sexo masculino que frequenta o ensino superior português. Método: Optou-se por uma abordagem exploratória quantitativa, a partir de uma amostra de 100 estudantes universitários do sexo masculino, que participaram num protocolo de investigação, constituído por um Questionário Sociodemográfico, Rosenberg Self-Esteem Scale (RSES) e Male Body Attitude Scale Revised (MBAS-R) (versões portuguesas) e pela medida do Índice de Massa Corporal. Respeitaram-se os procedimentos éticos das investigações em seres humanos. Resultados: Todos os instrumentos apresentam bons índices de consistência interna. A maioria dos participantes pratica exercício físico, tendo-se apurado um baixo grau de insatisfação com a imagem corporal e uma elevada autoestima. Os resultados apresentam uma relação negativa fraca, mas significativa entre a variável prática de exercício físico e classificação académica, não se verificando uma relação com as restantes variáveis. $\mathrm{O}$ grau de insatisfação com a imagem corporal apresenta diferenças estatisticamente significativas com a autoestima e classificação do IMC. Conclusões: Os participantes do estudo, apesar de mais de um terço apresentar peso acima do normal, percecionam maioritariamente baixo grau de insatisfação com a sua imagem corporal e apresentam elevada autoestima. Existe uma relação moderada entre o grau de insatisfação com a imagem corporal, o IMC e a autoestima nos estudantes da amostra. Os estudantes que detêm maior classificação académica praticam algum exercício físico. Não foram verificadas relações entre a prática de exercício físico e o IMC. O estudo evidencia a coexistência de estereótipos relativamente ao corpo ideal masculino.

Palavras-chave: Autoestima; Imagem corporal; Índice de Massa Corporal; Exercício físico

\begin{abstract}
Objective: This study aims to assess differences and possible relationships between the degree of dissatisfaction with body image, body mass index, practice of physical exercise, self-esteem, academic classification, and satisfaction with academic life in a sample of male students attending Portuguese higher education. Method: Opted for a quantitative exploratory approach, using a sample of 100 male university students, who participated in a research protocol consisting of a sociodemographic questionnaire, Rosenberg Self-Esteem Scale and Male Body Attitude Scale Revised (Portuguese versions) and the measurement of the body Mass Index. Ethical procedures for investigating human beings were respected. Results: The instruments have good rates of internal consistency. Most participants practice physical exercise, with a low degree of dissatisfaction with their body image and high self-esteem. The results show a weak but significant negative relationship between the variable of physical exercise and academic achievement, with no relationship with the other variables. The degree of dissatisfaction with body image presents statistically significant differences with self-esteem in relation to the BMI classification. Conclusions: The majority of participants to the study perceive a low degree of dissatisfaction with their body image and have good selfesteem, although more than a third are overweight. There is a moderate relationship between the degree of dissatisfaction with body image, BMI and self-esteem among the students in the sample. Students with higher levels of academic success practice some physical exercise. No relationship was found between the practice of physical exercise and BMI. The study shows the coexistence of stereotypes in relation to the ideal male body.
\end{abstract}

Keywords: Self-esteem, Body image, Body mass index, Physical exercise 


\section{INTRODUÇÃO}

As representações do corpo belo e saudável têm sofrido alterações ao longo dos tempos (Almeida et al., 2005; Bosi et al., 2006; Rodrigues et al., 2015), tal como nos evidenciam as representações iconográficas mais remotas, desde a antiguidade clássica, ou os escritos literários. Porém, desde os anos sessenta do século XX que se elege socialmente um corpo esbelto, magro, atlético, musculado e com formas esqueleticamente padronizadas (Almeida et al., 2005; Oliveira et al., 2003; Rodrigues et al., 2015).

Associado com a imagem de um corpo ideal/tipificado, tem emergido todo um mercado de produtos, muito aguerrido em estratégias de marketing, que impele a padrões de consumo, com vista a alcançar a imagem "perfeita", muitas vezes associada ao sucesso social como forma de poder, beleza e aceitação social (Rodrigues et al., 2015). Estes constructos socioculturais, muito projetados através das diferentes formas de comunicação e diferentes contextos (e.g., família, escolas, locais de entretenimento, redes sociais, etc.) vão influenciando a construção da imagem corporal das crianças, adolescentes, adultos emergentes (estudantes do ensino superior) e jovens adultos, nos seus processos desenvolvimentistas. A idealização corporal dos adolescentes e jovens pode conduzir a perceções erróneas do tamanho, formas e harmonia do corpo, levar a sentimentos de desvalorização e baixa autoestima, e conduzir à construção de uma imagem mental “inatingível” e não saudável (doente), afastando-se do corpo real.

Por imagem corporal entende-se a representação mental que cada indivíduo faz do seu próprio corpo e a forma como este é percebido e vivenciado (Carvalho et al., 2005; Rodrigues et al., 2015). Esta representação considera-se dinâmica, implica uma construção desenvolvimentista, e tem uma inter-relação com diferentes dimensões do desenvolvimento, mormente as dimensões física, psicossexual, afetiva, cognitiva e social. Price (1990) acrescenta uma dimensão plural do conceito de autoimagem corporal e especifica três autoimagens corporais - a real, a ideal e a social.

A maioria dos estudos que incidem sobre a preocupação com a imagem corporal têmse focado na avaliação da imagem corporal no sexo feminino (Almeida et al, 2005; Grogan, 2017) ou em estudos comparativos, em função do sexo. Rodrigues et al. (2015) evidenciam que, em adolescentes, a autoimagem difere em função dos sexos e que as perceções irrealistas do corpo são mais frequentes nas raparigas. $\mathrm{Na}$ verdade, são evidentes os estereótipos diferenciados em função dos sexos em relação ao corpo ideal, sendo que para o sexo feminino emerge frequentemente um corpo magro e com baixo percentual de gordura 
e para o sexo masculino um corpo atlético, musculado e com formas bem definidas (e.g., Carvalho et al., 2005; Fidelix et al., 2011; Rodrigues et al., 2015).

Estudos sobre a avaliação das preocupações com a imagem corporal no sexo masculino são escassos (Ferreira et al., 2018), no entanto, nos anos cinquenta, percebeu-se que os homens também se preocupam com possíveis alterações na imagem corporal, focando a sua atenção em algumas partes do corpo (e.g., cabelo, aptidão física e forma corporal) (Gay, 2009; Luciano, 2001). Segundo estes autores, existe um grupo de homens que pratica "bodybuilding" (construção de uma nova forma física com recurso a muito exercício físico e dieta apropriada) que tem como motivos o ganhar peso e a força, enquanto que outro grupo tem como objetivos impressionar as mulheres ou, ainda, procurar identificar-se com um dos seus ídolos (e.g., Arnold Schwarzenegger, Sylvester Stallone, G.I. Joe., entre outros).

A "ditadura" do corpo socialmente aceite gera, muito frequentemente, a insatisfação pessoal com a aparência. A insatisfação corporal nos homens associa-se ao desejo de ganhar massa muscular e perder gordura corporal (Reddy, 2013), idealizando um corpo musculoso, forte e robusto (Souza et al., 2013). Cash e Smolak (2012) revelam que quanto mais um jovem lê revistas, relacionadas com moda, saúde e boa forma masculina, maior é a probabilidade de se preocupar com a muscularidade e a condição física em idade adulta jovem, podendo recorrer ao uso de suplementos de musculação e esteroides, na intenção de conseguir o corpo desejado.

O estudo de Fawkner e McMurray (2002) revelou que as imagens de homens com corpos musculados apresentados nos meios de comunicação social são difíceis de serem ignoradas. A exposição dessas imagens pode assim contribuir para o aumento da insatisfação corporal no sexo masculino, predispondo-os a possíveis perturbações relacionadas com a imagem corporal (e.g., perturbações alimentares, uso de esteroides, dismorfia corporal e muscular) ou dismorfofobias.

Mendes et al. (2019) relatam que a valorização na gestão da aparência e a preocupação com a aparência no sexo masculino é inferior, quando comparado com o sexo feminino. Grogan (2017) refere que existe um consenso geral sobre a maioria dos homens ambicionar ter músculos bem definidos no peito, braços e ombros, isto é, preferem uma forma mesomórfica (constituição média) em vez de uma constituição ectomórfica (fina) ou endomórfica (gorda).

Estudos sobre a perceção da imagem corporal em estudantes universitários, avaliada com recurso ao Índice de Massa Corporal (IMC) como indicador do estado nutricional, revelam que a insatisfação com a imagem corporal se associa ao desejo de um IMC mais 
baixo (Barreto et al., 2019; Frank et al., 2016; Kakeshita \& Almeida, 2006; Lopes et al., 2017; Melo et al., 2016), e revelam que quanto maior for o IMC, menor é a prática de exercício físico por parte dos estudantes universitários (Primo et al., 2017; Vargas et al., 2016). Petry e Júnior (2019) também confirmaram tal ideia em praticantes de musculação, pois verificaram que, quanto maior o Índice de Massa Corporal e o peso corporal, maior era a insatisfação com a imagem corporal.

A atividade física pode ser considerada eficaz na redução da insatisfação com a imagem corporal (SantaBarbara et al., 2017) e contribuir diretamente para a apreciação corporal (Tylka \& Homan, 2015). Porém, Costa et al. (2014) defendem que os homens quando não se sentem tão musculados como gostariam, podem apresentar uma perceção mais negativa sobre a prática de exercício físico. Apesar de os homens que praticam exercício físico desejarem um corpo mais musculado (Melching et al., 2016), outros estudos referem que a aptidão física, a atratividade, a preocupação ou insatisfação com a sua aparência e o desejo de perder peso são outras das razões que levam os homens à prática de exercício físico (Fermino et al., 2010; Parmar \& Desai, 2015).

A relação entre a autoestima (entenda-se a estima que o indivíduo tem por si próprio) e a satisfação corporal tem sido estudada, evidenciando uma relação forte entre estas variáveis, ou seja, um nível elevado de satisfação corporal parece estar associado a uma elevada autoestima e vice-versa; ou seja, quanto maior a insatisfação com o corpo, mais baixa é a autoestima (Baker \& Blanchard, 2018; Cash \& Smolak, 2012; Kayani et al., 2018; Lin \& Lin, 2018; Putra et al., 2020; Robins et al., 2002; Romano et al., 2007, Santos e Maia, 2003). Por outro lado, parece existir uma relação entre imagem corporal e rendimento académico. Mendes e Medeiros (2019) levantam a hipótese de que preocupação com a imagem corporal possa ser um preditor de insucesso escolar.

A insatisfação com a imagem corporal parece ser um fenómeno comum entre os estudantes universitários (Souza \& Alvarenga, 2016), e sendo a investigação sobre a (in)satisfação com a imagem corporal com homens mais escassa, pretende-se com este estudo: (i) compreender a frequência da prática de exercício físico e o Índice de Massa Corporal em estudantes universitários do sexo masculino; (ii) verificar as relações e efeitos estatísticos entre o grau de (in)satisfação com imagem corporal e o rendimento académico; (iii) cotejar as relações e diferenças estatísticas entre os níveis de insatisfação com a imagem corporal, a autoestima, o rendimento académico e o IMC; e (iv) comparar os níveis de satisfação com a imagem corporal e a autoestima, através do ponto de corte de ambos os instrumentos. 


\section{MÉTODO}

Este estudo é de natureza exploratória descritiva, quantitativa e transversal com base numa amostra aleatória sistemática.

\subsection{Amostra}

A amostra constituiu-se por 100 estudantes universitários do sexo masculino, com idades compreendidas entre os 18 e os 44 anos (cf. Figura 1), com uma média de 22.89 anos de idade $(\mathrm{DP}=1.42)$. Os participantes frequentam os cursos de licenciatura $(\mathrm{n}=82)$, mestrado $(n=16)$ e doutoramento $(n=2)$ em universidades portuguesas. Os pais dos participantes detêm as habilitações académicas seguintes: ensino superior (14\%), ensino secundário (27\%), 3o ciclo do ensino básico (20\%), 2o ciclo do ensino básico (24\%) e 1o ciclo do ensino básico (14\%), enquanto que as mães dos participantes apresentam maior nível de habilitações literárias em relação aos pais dos participantes, a saber: ensino superior (30\%), ensino secundário (20\%), 3 ciclo do ensino básico (26\%), 2o ciclo do ensino básico (19\%) e $1^{\circ}$ ciclo do ensino básico $(5 \%)$.

\section{Figura 1}

Percentagem de idades dos participantes $(N=100)$

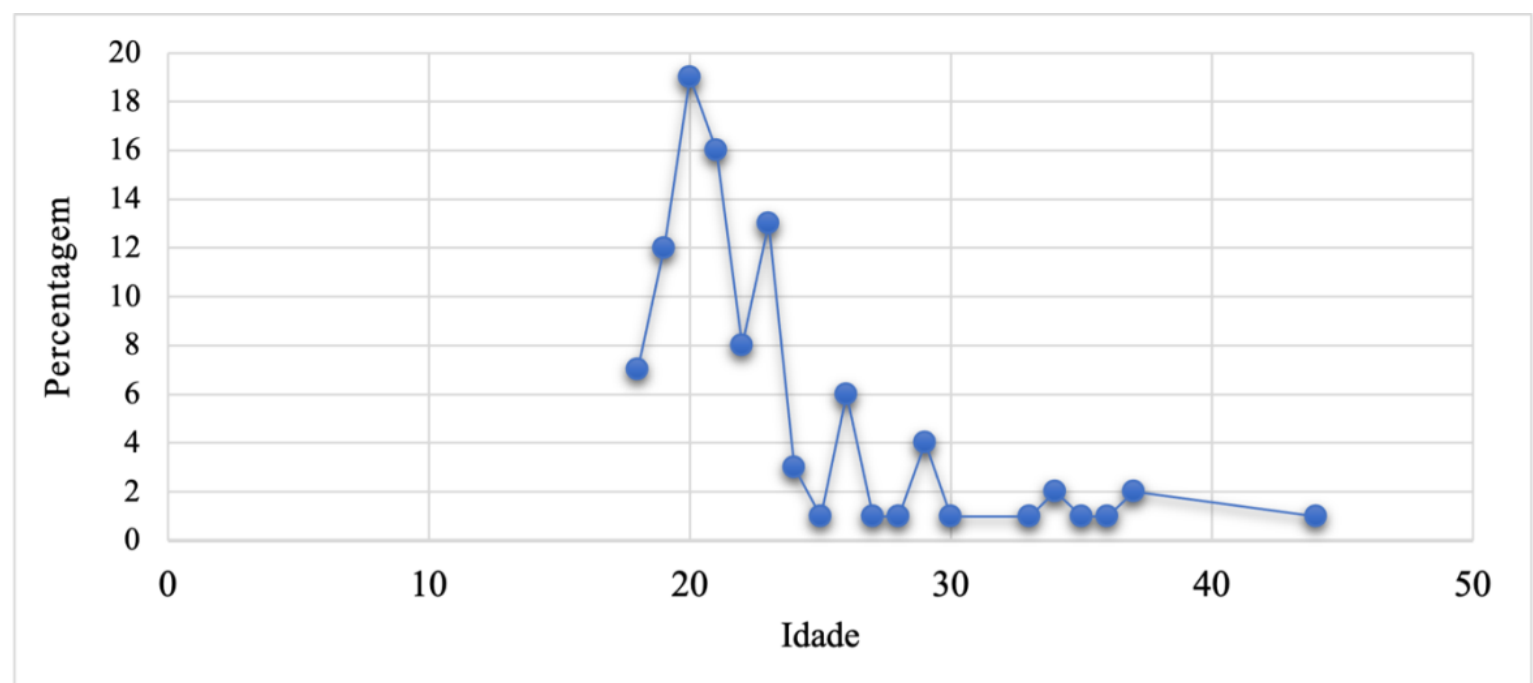

Os participantes referem estar maioritariamente satisfeitos com a vida académica (n =47), sendo que $10 \%$ estão muito satisfeitos. Os restantes estudantes estão medianamente satisfeitos $(n=37)$, insatisfeitos $(n=4)$ e muito insatisfeitos $(n=2)$.

\subsection{Instrumentos}

Para este estudo, optou-se por recorrer a vários instrumentos de medida, para medir as várias variáveis em análise, tal como abaixo se explicita. 
Elaborou-se um Questionário Sociodemográfico de forma a analisar as variáveis: idade, altura, peso, prática de exercício físico e respetiva modalidade, média de notas/classificações finais, grau de satisfação com a vida académica e nível de escolaridade dos pais.

Aplicou-se a Escala de Autoestima de Rosenberg (Rosenberg Self-Estime Scale) [RSES; versão original de Rosenberg (1965); versão portuguesa de Santos e Maia (2003)] para medir a variável autoestima. Trata-se de uma escala unidimensional de tipo Likert, constituída por 10 itens, com quatro opções gradativas de resposta, que vão desde o 1Concordo muito até ao 4- Discordo muito.

As atitudes e preocupações em relação à imagem corporal foram avaliadas através da Escala de Atitudes Corporais Masculinas - Versão Revista (Male Body Attitude Scale Revised) [MBAS-R; versão original de Tylka et al. (2005), na versão portuguesa de Ferreira et al. (2018)]. Trata-se de uma versão revista de um instrumento de medida, especificamente desenvolvido para a população masculina. A MBAS-R é composta por 12 itens divididos em duas subescalas: massa muscular (SMM) composta por 7 itens e massa gorda (SMG); com 5 itens. A escala, de tipo Likert, possibilita 6 pontos de resposta (1 - "Nunca", 2 - "Quase nunca", 3 - "Algumas vezes; 4 - "Muitas vezes", 5 - "Quase sempre" e 6 - "Sempre”). Pontuações mais elevadas da escala são indicadoras de maior insatisfação com a imagem corporal.

Mediu-se, ainda, o Índice de Massa Corporal [IMC; World Health Organization [WHO] (2000); Direção Geral de Saúde [DGS] (2005)]. Trata-se de uma classificação internacional que estima de forma simples se um indivíduo apresenta baixo peso, peso normal ou excesso de peso, através da fórmula, IMC $=\frac{K G}{m^{2}}$. Com base nesta classifica-se os indivíduos com baixo peso quando o IMC é inferior a 18.5; peso normal quando apresenta valores entre 18.5-24.9; pré-obesidade entre 25-29.9. A medida permite também enquadrar os três tipos de obesidade: classe I, 30-34.9; classe II, 35-39.9 e classe III $\geq 40$.

\subsection{Procedimentos}

Foram solicitadas as autorizações para a aplicação dos instrumentos aos estudantes, procedendo-se à elaboração do questionário de caracterização sociodemográfica. Posteriormente, reuniram-se todos os instrumentos num protocolo, acompanhados do Termo de Consentimento Livre e Esclarecido. Os instrumentos foram disponibilizados aos participantes presencialmente (formato papel) e através da plataforma digital (Google 
Forms), tendo sido divulgados através de $e$-mail e redes sociais (e.g., facebook), a fim de se conseguir maior abrangência da amostra.

Como critérios de inclusão no estudo, os participantes tinham de ser do sexo masculino, ter mais de 18 anos, frequentar o ensino universitário e desejar participar de forma livre e voluntária. Obtiveram-se no total 101 respostas, recolhidas através de uma plataforma digital $(n=32)$ e aplicação do questionário presencialmente $(n=69)$. Eliminouse um questionário, por não respeitar o critério de inclusão "ser do sexo masculino".

\subsection{Análise Estatística}

Para a realização dos procedimentos estatísticos, recorreu-se ao software SPSS 25 para Macintosh e AMOS 21 para Windows. Determinou-se a fiabilidade dos instrumentos pela análise dos coeficientes do alfa de Cronbach $(\alpha)$. Procedeu-se à análise descritiva com recurso a medidas de localização (cálculo da média total dos instrumentos com recurso ao ponto de corte, dispersão e associação); à análise dos coeficientes de correlação de Pearson (r) para quantificar a intensidade e direção da associação entre as variáveis; e à aplicação dos testes $t$-Student e ANOVA com o respetivo tamanho do efeito (TDE) (Espírito-Santo \& Daniel, 2015; Marôco, 2018), de forma a verificar diferenças estatísticas.

\section{RESULTADOS}

Após análise da fiabilidade das escalas, verificou-se que os níveis de consistência interna foram considerados moderados a elevados [.8 - .9] (Marôco \& Garcia-Marques, 2006) para todos os instrumentos [RSES $(\alpha=.87)$; MBAS-R $(\alpha=.85)]$. As subescalas que compõem o instrumento MBAS-R, apresentam um alfa de Cronbach de moderado a elevado $(\alpha=.84)$ para a subescala massa muscular [SMM] e um alfa de Cronbach elevado $(\alpha=.90)$ para a subescala massa gorda [SMG].

Para verificar a frequência da prática do exercício físico e o índice de massa corporal procedemos, inicialmente, a uma análise descritiva simples. Assim, verificou-se que a maioria dos participantes (58\%) pratica exercício físico. Dentro dos que praticam exercício físico, a maioria frequenta o ginásio (e.g., exercícios do tipo musculação, fitness, kickboxing), seguindo-se desportos relacionados com a bola (e.g., futebol, andebol, voleibol e ténis) e $15 \%$ indicam que fazem exercícios de cardiovascular, ou seja, exercícios do tipo de corrida e caminhada (Figura 2). Não se pode deixar de destacar que $42 \%$ dos estudantes não praticam exercício físico, o que pode condicionar um estilo de vida saudável. 


\section{Figura 2}

Modalidade da Prática de Exercício Físico (n=58)

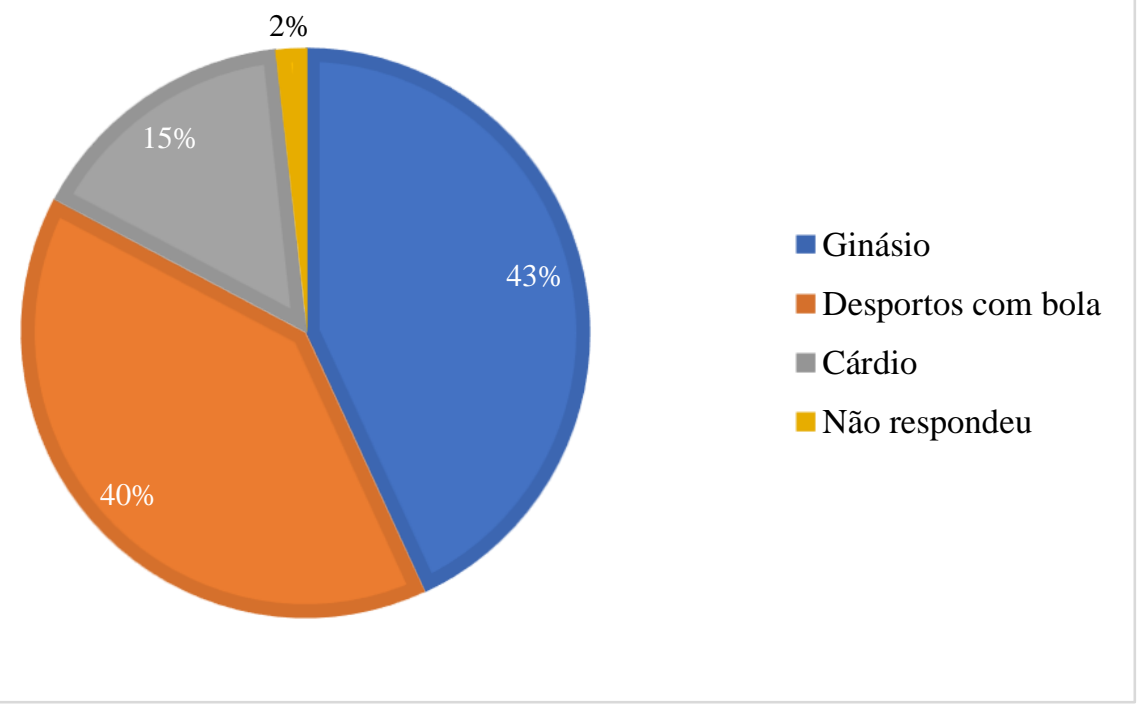

Na verificação do Índice de Massa Corporal também 58\% dos estudantes apresentam quer peso adequado (IMC entre $>18.5$ e $\geq 25$ ), quer sobrepeso (28\%, com IMC entre $\geq 25$ e $<30$ ). Verificou-se que $4 \%$ da amostra apresenta baixo peso (IMC $<18.5$ ), tendo-se verificado que $9 \%$ apresentam obesidade classe I (IMC entre $\geq 30$ e $<35$ ). Somente um participante apresenta obesidade severa - classe II (IMC entre $\geq 35$ e < 40) (DSG, 2005). No entanto não verificaram relações entre a prática de exercício físico e o IMC $(r=.015 ; p=$ $.88)$.

Para compreender a relação e o efeito entre a variável grau de insatisfação com a imagem corporal e respetivas subescalas (SMM e SMG), prática de exercício físico, grau de satisfação com a vida académica, autoestima, classificação académica e índice de massa corporal, fomos determinar as correlações bivariadas (cf. Tabela 1).

A análise das relações entre a variável grau de insatisfação com a imagem corporal e autoestima, revelou que as atitudes negativas dos participantes em relação à imagem corporal [MBAS-R] apresentam uma relação negativa moderada e significativa com a variável autoestima, verificando-se o mesmo para as dimensões Massa Muscular (SMM) e Massa Gorda (SMG). O grau de insatisfação com a imagem corporal e massa gorda apresenta uma relação moderada positiva e significativa entre a variável IMC. A variável grau de satisfação com a vida académica apresenta uma relação negativa fraca e significativa com a variável perceção de massa gorda [SMG], e uma relação moderada positiva e significativa 
com a variável autoestima [RSES], isto é, à medida que aumenta a satisfação com a vida académica diminui a perceção de massa gorda e aumenta a autoestima. Os resultados apresentam uma relação negativa fraca, mas significativa entre a variável prática de exercício físico e classificação académica.

\section{Tabela 1}

Correlações Bivariadas entre os Instrumentos Male Body Atitude Scale respetivas Subescalas (Massa Muscular, Massa Gorda), Rosenberg Self-Estime Scale e as Variáveis Sociodemográficas (Prática de Exercício Físico, Grau de Satisfação com a Vida Académica, Classificação Académica e Índice de Massa Corporal)

\begin{tabular}{lcccccccc}
\hline & MBAS-R & SMM & SMG & RSES & PEF & GSVA & CA & IMC \\
\hline MBAS-R & -- & $.814^{* *}$ & $.775^{* *}$ & $-.486^{* *}$ & .080 & -.165 & -0.065 & $.319^{* *}$ \\
\multicolumn{1}{c}{ SMM } & & -- & $.263^{* *}$ & $-.378^{* *}$ & .169 & -.053 & -.131 & -.037 \\
SMG & & & -- & $-.395^{* *}$ & -.050 & $-.216^{*}$ & .035 & $.569^{* *}$ \\
RSES & & & & -- & -.132 & $.424^{* *}$ & .180 & -.031 \\
PEF & & & & & -- & .056 & $-.235^{*}$ & -.20 \\
GSVA & & & & & & -- & .182 & .090 \\
CA & & & & & & & - & .147 \\
IMC & & & & & & & & - \\
\hline M & 30.36 & 19.73 & 10.73 & 33.17 & 1.42 & 3.59 & 2.60 & 24.30 \\
DP & 9.82 & 6.43 & 5.92 & 5.70 & .49 & .80 & .86 & 4.03 \\
\hline
\end{tabular}

Nota: MBAS-R = Male Body Atitude Scale Revised; SMM = Subescala Massa Muscular; SMG = Subescala Massa Gorda; RSES = Rosenberg Self-Estime Scale; PEF = Prática de Exercício Físico; GSVA = Grau de Satisfação com a Vida Académica; CA = Classificação Académica; IMC = Classificação do Índice de Massa Corporal (em classes); M = Média; DP = Desvio Padrão. $* p=.05 ; * * p=.01$

Por meio do teste $t$-Student, verificaram-se diferenças estatisticamente significativas na variável classificação académica em função da prática de exercício físico (cf. Tabela 2), com um TDE pequeno $(g$ de Cohen $=.48)($ Espírito-Santo \& Daniel, 2015). A prática de exercício físico não teve efeitos estatisticamente significativos nas restantes variáveis.

Tabela 2

Diferenças da Classificação Académica Entre a Prática de Exercício Físico (n=100)

\begin{tabular}{|c|c|c|c|c|c|c|c|c|}
\hline \multirow{2}{*}{ Variáveis } & \multirow{2}{*}{$\begin{array}{l}\text { Prática de exercício } \\
\text { físico }\end{array}$} & \multirow{2}{*}{$n$} & \multirow{2}{*}{$M$} & \multirow{2}{*}{$D P$} & \multirow{2}{*}{$t$} & \multirow{2}{*}{$p$} & \multicolumn{2}{|c|}{$95 \% I C$} \\
\hline & & & & & & & Inferior & Superior \\
\hline \multirow{2}{*}{ Classificação académica } & Sim & 57 & 2.77 & .89 & \multirow{2}{*}{2.36} & \multirow{2}{*}{.20} & \multirow{2}{*}{.065} & \multirow{2}{*}{.747} \\
\hline & Não & 41 & 2.37 & .77 & & & & \\
\hline
\end{tabular}

Relativamente às diferenças estatísticas, verificamos que o grau de satisfação com a vida académica teve um efeito estatisticamente significativo e de elevada dimensão $(F(4,95)$ $\left.=6.908 ; p=<.001 ; \eta_{p}^{2}=.225 ; \pi=.99\right)$ sobre a variável autoestima, não existindo diferenças 
estatisticamente significativas com as restantes variáveis. O IMC apresenta diferenças estatisticamente significativas com os níveis de insatisfação com a imagem corporal (MBAS-R), a variável massa gorda (SMG) e a variável autoestima (RSES) (cf. Tabela 3). Através do recurso ao teste Post-hoc HDS de Games-Howell, as diferenças estatisticamente significativas para a variável insatisfação com a imagem corporal foram encontradas entre a classificação do índice de massa corporal "Obesidade" e "Variação normal" (IC 95\% ]1.25; 18.11[ $p=.018$ ); para a variável massa gorda foram encontradas entre "Pré-obesidade" $\mathrm{e}$ "Baixo peso" (IC 95\%]1.18; 15.31[ $p=.015$ ), "Pré-obesidade" e "Variação normal" (IC 95\% ]1.82; $7.90[p=<.001$ ), "Obesidade" e "Baixo peso" (IC 95\% ]4.23; $19.87[p=.001$ ) e "Obesidade" e "Variação normal" (IC 95\% ]4.13; 13.19 [ $p=<.001$ ). Se se tiver em consideração a correção de Bonferroni $(p<.002)$, a diferença entre a variável autoestima e classificação do índice de massa corporal perde significância estatística (Pallant, 2010).

Tabela 3

Diferenças em Relação às Variáveis Insatisfação com a Imagem Corporal (MBAS-R, SMM, SMG) $e$ Autoestima (RSES) entre Participantes com Diferentes Classificações do Índice de Massa Corporal

\begin{tabular}{|c|c|c|c|c|c|c|c|c|}
\hline \multirow{2}{*}{ Classificação do IMC } & \multirow[b]{2}{*}{$n$} & \multirow[b]{2}{*}{$M(D P)$} & \multicolumn{4}{|c|}{ ANOVA } & \multicolumn{2}{|c|}{$95 \%$ IC } \\
\hline & & & $F(g l)$ & $p$ & $\eta_{p}^{2}$ & $\pi$ & Inferior & Superior \\
\hline MBAS-R & & & 3.94(96) & .011 & .110 & .818 & & \\
\hline Baixo Peso & 4 & $27.25(7.41)$ & & & & & 15.46 & 39.04 \\
\hline Variação Normal & 58 & $28.22(8.41)$ & & & & & 26.01 & 30.43 \\
\hline Pré-obesidade & 28 & $32.89(9.82)$ & & & & & 29.09 & 36.70 \\
\hline Obesidade & 10 & $37.90(9.83)$ & & & & & 28.01 & 47.79 \\
\hline SMM & & & $.26(96)$ & .857 & .008 & .097 & & \\
\hline Baixo Peso & 4 & $22(7.26)$ & & & & & 10.45 & 33.55 \\
\hline Variação Normal & 58 & $19.59(6.63)$ & & & & & 17.84 & 21.33 \\
\hline Pré-obesidade & 28 & $10.39(5.92)$ & & & & & 17.10 & 21.69 \\
\hline Obesidade & 10 & $20.60(7.12)$ & & & & & 15.51 & 25.69 \\
\hline SMG & & & $13.31(96)$ & $<.001$ & .295 & 1 & & \\
\hline Baixo Peso & 4 & $5.25(.50)$ & & & & & 4.45 & 6.05 \\
\hline Variação Normal & 58 & $8.64(4.65)$ & & & & & 7.42 & 9.86 \\
\hline Pré-obesidade & 28 & $13.50(4.79)$ & & & & & 11.64 & 15.36 \\
\hline Obesidade & 10 & $17.30(8.18)$ & & & & & 11.45 & 23.15 \\
\hline Autoestima & & & $2.74(96)$ & .047 & .079 & .648 & & \\
\hline Baixo Peso & 4 & $27.25(5.06)$ & & & & & 19.20 & 35.30 \\
\hline Variação Normal & 58 & $33.97(4.84)$ & & & & & 32.69 & 35.24 \\
\hline Pré-obesidade & 28 & $33.36(5.97)$ & & & & & 31.04 & 35.67 \\
\hline Obesidade & 10 & $30.40(8.13)$ & & & & & 24.59 & 36.21 \\
\hline
\end{tabular}

Por fim, e considerando o ponto de corte para cada um dos instrumentos, os resultados revelam que $83 \%$ apresentam uma elevada autoestima $(M=33.17 ; D P=5.71) \mathrm{e}$ 91\% dos participantes apresentam graus inferiores de insatisfação com a imagem corporal 
$(M=30.46 ; D P=9.83)$, isto é, percecionam alguns índices de satisfação com a sua imagem corporal. No entanto, realçamos que apesar de os participantes em estudo apresentarem graus inferiores de insatisfação com a imagem corporal, 38\% dos participantes apresentam um peso acima do normal $(\mathrm{IMC}=]>25 ; 40[$ ).

\section{DISCUSSÃO}

Os instrumentos aplicados apresentam uma consistência interna moderada a elevada $(\alpha>$ 0.80), revelando-se indicadores de boa confiabilidade para os fatores avaliados (Marôco \& Garcia-Marques, 2006).

Os resultados, apresentam uma relação entre o grau de insatisfação com imagem corporal e o IMC, existindo maior relação e com maior significância quando se refere à subescala de massa gorda. O presente estudo vai ao encontro dos resultados apresentados por Ferreira et al. (2018), verificando a existência de associações positivas e significativas com a MBAS-R e a subescala massa gorda e o IMC. Apesar de, também se ter verificado uma associação negativa entre o IMC e a subescala massa muscular, esta associação não se apresentou significativa, ao contrário do resultado apresentado aquando a validação do MBAS-R. Por outro lado, um estudo realizado em 2013 por Souza et al. avaliou o corpo na perspetiva masculina e revelou que, apesar de não existir uma relação entre o IMC com a satisfação corporal, valores extremos podem gerar maior insatisfação com o corpo. Este estudo vai no mesmo sentido dos nossos resultados, uma vez que se verificou uma relação significativa entre o grau de insatisfação com a imagem corporal e o IMC, existindo diferenças estatisticamente significativas entre os extremos do IMC ("Baixo peso" e "Obesidade").

Por outro lado, $90 \%$ dos participantes neste estudo apresentam um baixo grau de insatisfação com a imagem corporal, apesar de 38\% dos participantes revelarem um peso acima da variação normal. Neste sentido, um estudo realizado por Talbot et al. (2019) revela que a massa gorda e a musculosidade não se associam à insatisfação com a imagem corporal.

Os resultados verificam que a autoestima não apresenta uma relação significativa com o IMC, pois apesar de se verificarem diferenças estatisticamente significativas entre as variáveis, essas diferenças perdem significância, apresentando médias muito próximas entre na classificação do IMC. No entanto, um estudo realizado por Alvani et al. (2016), revelam que os estudantes do sexo masculino, têm maior tendência a um peso normal e que existe uma forte correlação entre a autoestima e o IMC. Estes autores defendem ainda que quanto maior a autoestima, maior a probabilidade de apresentar uma variação do peso normal. 
Outros estudos expressaram que a prática de exercício físico influencia o aumento da autoestima (Gilani \& Dashipour, 2017; Moral-García et al., 2018), no entanto, os nossos resultados não evidenciam relações e diferenças estatisticamente significativas entre a prática de exercício físico, autoestima e o grau de insatisfação com a imagem corporal. No mesmo sentido Machado et al. (2019), revelam não existir uma relação entre a prática de exercício físico, a autoestima e o IMC, enquanto que Leng et al. (2020) concluem não existirem diferenças significativas entre a satisfação com a imagem corporal e prática de exercício físico no sexo masculino.

Através dos resultados apresentados, verificou-se também a existência de uma fraca relação significativa entre a prática de exercício físico e a classificação académica, isto é, os participantes que praticavam exercício físico apresentam uma classificação académica superior aos que não praticavam exercício físico, indo ao encontro de estudos que verificaram a influência da prática de exercício físico no desempenho académico dos estudantes (Batista et al., 2016; Kayani et al., 2018).

Ao avaliar possíveis relações e diferenças estatísticas entre a classificação académica dos participantes e o grau de insatisfação com a imagem corporal, os resultados não evidenciaram diferenças estatisticamente significativas, reforçando o estudo de Mendes e Medeiros (2019) que revela a variável preocupação com a imagem corporal como possível preditor de (in)sucesso académico.

Pelo ponto de corte, verifica-se que a maioria dos participantes apresenta graus inferiores de insatisfação com a imagem corporal e apresentam elevada autoestima. Observou-se que quanto menor o grau de insatisfação com a imagem corporal maior a autoestima dos participantes. Lin e Lin (2018) verificaram, no seu estudo, uma forte relação entre a perceção positiva da imagem corporal e os altos níveis de autoestima e Qaisy (2016) encontrou baixos níveis de autoestima e insatisfação com a imagem corporal em estudantes universitários, existindo uma relação positiva entre a imagem corporal e a autoestima.

\section{CONCLUSÕES}

No último século, particularmente após a segunda metade do século $\mathrm{XX}$, que tem vindo a emergir, em geral, a eleição por um corpo magro e atlético. Para esta representação muito têm contribuído os meios de comunicação social, em especial a televisão (e.g., através dos filmes, das telenovelas, dos concursos de beleza e da publicidade), a imprensa escrita (e.g., as revistas de moda e de eventos sociais), a indústria da moda, o marketing e, mais recentemente, as redes sociais que, no seu conjunto, têm exercido uma grande influência na 
(in)satisfação com a imagem corporal e na projeção de um corpo padronizado em função dos sexos. Nesta sequência, o conceito de imagem corporal tem sido alvo de investigação nas últimas décadas, tendo-se percebido que a construção da imagem corporal pode modificar-se ao longo do tempo.

A investigação revela que coexistem os estereótipos relativamente ao corpo ideal e à imagem perfeita, em função dos sexos, atribuindo um corpo magro e com baixa percentagem de gordura para o sexo feminino e um corpo atlético, musculado e com formas esqueléticas bem definidas para o sexo masculino (e.g., corpo em V). Os estudos sobre a imagem corporal no sexo masculino são escassos e carecem de maior profundidade, nas suas relações com variáveis psicológicas e com as diferentes dimensões do desenvolvimento.

Apesar da amostra de estudantes do sexo masculino a frequentar o ensino superior se revelar pequena neste estudo - uma centena de estudantes - os resultados, permitem concluir que existe uma mediana adesão à prática de exercício físico nos estudantes, sendo os estudantes que praticam exercício físico preferirem, em primeiro lugar, frequentar o ginásio, com os exercícios habituais nestes locais (exercícios do tipo musculação, fitness, kickboxing), em segundo lugar os exercícios "com a bola" (entenda-se o futebol) e, ainda, numa percentagem muito pouco expressiva da amostra, os exercícios de cardiovascular (sendo alguns exemplos a corrida, a caminhada e a natação).

Conclui-se nesta amostra que a variável autoestima apresenta uma associação positiva com a variável satisfação com a vida académica e uma associação negativa com o grau de insatisfação com a imagem corporal. Além disso, os participantes que praticam exercício físico apresentam maior sucesso académico dos que não praticam exercício físico, não se verificando nenhuma relação entre a prática de exercício físico e as restantes variáveis (e.g. insatisfação com a imagem corporal, subescala massa magra, subescala massa gorda, classificação académica e índice de massa corporal). É também possível concluir que a baixa perceção do grau de insatisfação com a imagem corporal está moderadamente relacionada com uma maior autoestima, enquanto que o grau de insatisfação com a imagem corporal se relaciona com um índice de massa corporal adequado.

Limitações no presente estudo devem ser tidas em consideração, dado que as respostas através da plataforma digital não garantem que todos os participantes sejam efetivamente do sexo masculino e não se ter explorado há quanto tempo é que os participantes praticam exercício físico. Outras limitações inerentes ao estudo referem-se ao facto de não se ter explorado o uso das redes sociais (e.g.facebook, instagram, tinder, grindr, outras) pelos participantes em estudo. Sugerem-se futuros estudos que avaliem possíveis 
relações entre imagem corporal, comportamentos alimentares (e.g., tipo de alimentação, dietas), hábitos de vida saudável e outros métodos adicionais para avaliar a composição do corpo para melhor interpretar a classificação do IMC. Apesar destas limitações cremos ter dado um contributo para o estudo da imagem corporal e a autoestima em homens estudantes.

Conflitos de interesse: Os autores declaram a inexistência de conflitos de interesse na realização do trabalho. Dois autores participam na equipa Editorial, mas não desempenharam qualquer papel de intervenção no processo de revisão por pares double-blinded.

Fontes de financiamento: Não existem fontes externas de financiamento para a realização deste artigo.

Agradecimentos: Os autores não têm quaisquer apoios a declarar.

\section{Referências}

Almeida, G., Santos, J. Pasian, S., \& Loureiro, S. (2005). Perceção de tamanho e forma corporal de mulheres: Estudo exploratório [Perceptions of body shape and size in women: An exploratory study]. Psicologia em Estudo, 10(1), 27-35. http://www.scielo.br/pdf/pe/v10n1/v10n1a04.pdf

Alvani, S. R., Mehrshad, S., Hosseini, P., \& Kimura, L. W. (2016). Relationship between body weight and self-esteem: A study of young men and women in Iran. Journal of Obesity and Overweight, 2(2), 1-8. https://doi.org/10.15744/2455-7633.2.202

Baker, A., \& Blanchard, C. (2018). Men's body image: The effects of an unhealthy body image on psychological, behavioral, and cognitive health. In I. J. Lobera (Ed.), Weight Loss (pp. 101119). InTech. https://doi.org/10.5772/intechopen.75187

Barreto, J. T. T., Rendeiro, L. C., Nunes, A. R. M., Ramos, E. M. L. S., Ainett, W. S. O., LourençoCosta, V. V., \& Sá, N. N. B. (2019). Fatores associados à insatisfação com a imagem corporal em estudantes dos cursos da área da saúde de Belém-PA [Factors associated with dissatisfaction with body image in students of health courses in Belém-PA]. Revista Brasileira de Obesidade, Nutrição e Emagrecimento, 13(77), 120-128. http://www.rbone.com.br/index.php/rbone/article/view/894

Batista, M., Cubo, D. S., Honório, S., \& Martins, J. (2016). The practice of physical activity related to self-esteem and academical performance in students of basic education. Journal of Human Sport and Exercise, 11(2), 297-310. https://doi.org/10.14198/jhse.2016.112.03

Bosi M. L. M., Luiz, R. R., Morgado, C. M. C., Costa, M. L., \& Carvalho, R. J. (2006). Autopercepção da imagem corporal entre os estudantes de nutrição: Um estudo no município do Rio de Janeiro [Self-perception of body image among nutrition students: a study in the city of Rio de Janeiro]. Jornal Brasileiro de Psiquiatria, 55(2), 108-113. https://doi.org/10.1590/S0047-20852006000200003.

Carvalho, A. M. P., Cataneo, C., Galindo, E. M. C., \& Malfará, C. T. (2005). Auto-conceito e imagem corporal em crianças obesas [Self concept and body image in obese children]. Paidéia, 15(30), 131-139. https://doi.org/10.1590/S0103-863X2005000100014.

Cash, T., \& Smolak, L. (2012). Body image: A handbook of science, practice, and prevention ( $2^{\mathrm{a}}$ Ed). The Guilford Press.

Costa, A., Araújo, A., Diniz, A., \& Almeida, L. (2014). Expetativas académicas segundo o género dos estudantes portugueses do primeiro ano do ensino superior. International Journal of 
Developmental and Educational Psychology, 2(1), 325-334. http://www.infad.eu/RevistaINFAD/OJS/index.php/IJODAEP/article/view/447/374

Diener, E., Emmons, R. A., Larsen, R. J., \& Griffin, S. (1985). The satisfaction with life scale. Journal of Personality Assessment, 49(1), 71-75. https://doi.org/10.1207/s15327752jpa4901_13

Direção Geral de Saúde. (2005). Programa nacional de combate à obesidade. Direção Geral de Saúde. $\quad$ https://www.dgs.pt/areas-em-destaque/plano-nacional-de-saude/programasnacionais/programa-nacional-de-combate-a-obesidade.aspx

Espírito-Santo, H., \& Daniel, F. B. (2015). Calcular e apresentar tamanhos do efeito em trabalhos científicos (1): As limitações do $\mathrm{p}<0,05$ na análise de diferenças de médias de dois grupos [Calculating and reporting effect sizes on scientific papers (1): $p<0.05$ limitations in the analysis of mean differences of two groups]. Revista Portuguesa de Investigação Comportamental e Social, 1(1), 3-16. https://doi.org/10.7342/ismt.rpics.2015.1.1.14

Fawkner, H., \& McMurray, N. (2002). Body image in men: Self-reported thoughts, feelings, and behaviors in response to media images. International Journal of Men's Health, 1(2), 137162. https://doi.org/10.3149/jmh.0102.137

Fermino, R. C., Pezzini, M. R., \& Reis, R. S. (2010). Motivos para prática de atividade física e imagem corporal em frequentadores de academia [Reasons for physical activity practice and body image among health clubs users]. Revista Brasileira de Medicina do Esporte, 16(1), 18-23. https://doi.org/10.1590/S1517-86922010000100003

Ferreira, C., Marta-Simões, J., Oliveira, S., \& Duarte, J. (2018). Estudo da estrutura fatorial e das qualidades psicométricas da versão portuguesa da Male Body Attitude Scale-Revised [Male Body Attitudes Scale-Revised: Factor structure and psychometric properties of the Portuguese version]. Revista Portuguesa de Investigação Comportamental e Social, 4(2), 16-24. https://doi.org/10.31211/rpics.2018.4.2.74

Fidelix, Y., Silva, D., Pelegrini, A., Silva, A., \& Petroski, E. (2011). Insatisfação com a imagem corporal em adolescentes de uma cidade de pequeno porte: associação com sexo, idade e zona de domicílio [Body image dissatisfaction among adolescents from a small town: Association with gender, age, and area of residence]. Revista Brasileira de Cineantropometria \& Desempenho Humano, 13(3), 202-207. https://doi.org/10.5007/19800037.2011v13n3p202.

Frank, R., Claumann, G. S., Pinto, A. de A., Cordeiro, P. C., Felden, É. P. G., \& Pelegrini, A. (2016). Fatores associados à insatisfação com a imagem corporal em acadêmicos de Educação Física [Factors associated to body image dissatisfaction in Physical Education college students]. Jornal Brasileiro de Psiquiatria, 65(2), 161-167. https://doi.org/10.1590/00472085000000118

Gay, K. (2009). Body image and appearance: The ultimate teen guide. Scarecrow Press.

Gilani, S. R. M., \& Dashipour, A. (2017). The effects of physical activity on self-esteem: A comparative study. International Journal of High Risk Behaviors and Addiction, 6(1), 1-6. https://doi.org/10.5812/ijhrba.35955

Grogan, S. (2017). Body image: Understanding body dissatisfaction in men, women and children ( $3^{\mathrm{a}}$ Ed). Routledge.

Kakeshita, I. S., \& Almeida, S. S. (2006). Relação entre índice de massa corporal e a percepção da auto-imagem em universitários [Relationship between body mass index and self-perception among university students]. Revista de Saúde Pública, 40(3), 497-504. https://doi.org/10.1590/S0034-89102006000300019

Kayani, S., Kiyani, T., Wang, J., Zagalaz Sánchez, M., Kayani, S., \& Qurban, H. (2018). Physical activity and academic performance: The mediating effect of self-esteem and depression. Sustainability, 10(10), 1-17. https://doi.org/10.3390/su10103633 
Leng, H. K., Phua, Y. X. P., \& Yang, Y. (2020). Body image, physical activity and sport involvement: A study on gender differences. Physical Culture and Sport. Studies and Research, 85(1), 40-49. https://doi.org/10.2478/pcssr-2020-0005

Lin, H.-C., \& Lin, Y.-C. (2018). The study of body image, self-esteem and sexual satisfaction of college students in southern Taiwan. Universal Journal of Educational Research, 6(4), 647652. https://doi.org/10.13189/ujer.2018.060408

Lopes, M. A. M., Paiva, A. A., Lima, S. M. T., Cruz, K. J. C., Rodrigues, G. P., \& Carvalho, C. M. R. G. (2017). Percepção da imagem corporal e estado nutricional em acadêmicas de nutrição de uma universidade pública [Perception of body image and nutritional status in nutrition undergraduates from a public university]. DEMETRA: Alimentação, Nutrição \& Saúde, 12(1), 193-206. https://doi.org/10.12957/demetra.2017.22483

Luciano, L. (2001). Looking good: Male body image in modern America. Hill and Wang.

Machado, A., Almeida, A., Fonte, D., \& Silva, V. (2019). Influência do exercício físico na autoestima e no índice de massa corporal [The influence of physical exercise on self-esteem and the body mass índex]. Psychtech \& Health Journal, 3(1), 19-29. https://doi.org/10.26580/PTHJ.art18-2019

Marôco, J., \& Garcia-Marques, T. (2006). Qual a fiabilidade do alfa de Cronbach? Questões antigas e soluções modernas? Laboratório de Psicologia, 4(1), 65-90. http://publicacoes.ispa.pt/index.php/lp/article/viewFile/763/706

Marôco, J. (2018). Análise estatística com o SPSS Statistics v18-25. ReportNumber

Melching, K. L., Green, J. M., O’Neal, E. K., \& Renfroe, L. (2016). Body image dissatisfaction: Responses between male and female exercisers and non-exercisers. International Journal of Exercise Science, 9(3), 249-257. https://core.ac.uk/download/pdf/43653017.pdf

Melo, P. E., Barcelos, S. C., Manochio, M. D. P. G., Vassimon, H. S., \& Bittar, C. M. L. (2016). Percepção da autoimagem corporal de universitários [Body self-image perception of university students]. Cinergis, 17(3), 1-6. https://doi.org/10.17058/cinergis.v17i3.7760

Mendes, J., \& Medeiros, T. (2019). Imagem corporal positiva em estudantes do ensino superior. In I. G. S. Arioli (Orgs), Psicologia da Saúde: Teoria e Intervenção (1. a ed.) (pp. 94-107). Ponta Grossa: Atena Editora. Confrontar em https://doi.org/10.22533/at.ed.7011912037

Mendes, J., Rego, R., \& Pereira, V. (2019). Tradução e adaptação da escala CARSAL/CARVAL para Portugal: Estudo psicométrico [Translation and Adaptation of the CARSAL / CARVAL Scale to Portugal: a psychometric study]. Revista da Associação Portuguesa de Psicologia, 33(1), 47-54. https://doi.org/10.17575/rpsicol.cv33i1.1425

Moral-García, J. E., García, D. O., García, S. L., Jiménez, M. A., \& Dios, R. M. (2018). Influencia de la actividad física en la autoestima y riesgo de dependencia en personas mayores activas y sedentárias [Influence of physical activity on self-esteem and risk of dependencein active and sedentary elderly people]. Anales de Psicología, 34(1), 162-166. https://doi.org/10.6018/analesps.34.1.294541

Oliveira, P. F., Bosi, M. L. M., Santos, P., \& Vieira, S. R. (2003). Eating behavior and body image in athletes. Revista Brasileira de Medicina do Esporte, 9(6), 357-364. https://doi.org/10.1590/S1517-86922003000600002.

Pallant, J. (2010). SPSS survival manual: A step by step guide to data analysis using SPSS for Windows $\left(4^{\mathrm{a}} \mathrm{ed}\right)$. Allen \& Unwin.

Parmar, S., \& Desai, M. (2015). Body weight effect on body image among gym users and non-gym users. The International Journal of Indian Psychology, 3(1), 88-94. http://oaji.net/articles/2015/1170-1449649464.pdf 
Petry, N. A., \& Júnior, M. P. (2019). Avaliação da insatisfação com a imagem corporal de praticantes de musculação em uma academia de São José-JC. Revista Brasileira de Nutrição Esportiva, 13(78), 219-226. http://www.rbne.com.br/index.php/rbne/article/view/1323/877

Primo, S. H., Molari, M., Luna, A. A., Bispo, N. N. C., Ossada, V. A. Y, \& Costa, V. S. P. (2017). Avaliação dos níveis de atividade física em estudantes universitários do ensino a distância [Evaluation of the Level of Physical Activity in University Students of Distance Education]. Journal of Health Sciences, 19(3), 143-148. https://doi.org/10.17921/24478938.2017v19n3p143-148

Price, B. (1990). A model for body-image care. Journal of Advanced Nursing, 15(5), 585-593. https://doi.org/10.1111/j.1365-2648.1990.tb01858.x

Putra, A., Sudiana, I. K., \& Giri, M. K. W. (2020). Interaction between body image and exercise activity. Proceedings of the 3rd International Conference on Innovative Research Across Disciplines (ICIRAD 2019). Denpansar, Indonesia. https://doi.org/10.2991/assehr.k.200115.071

Qaisy, L. (2016). Body image and self-esteem among ISRA' university students. British Journal of Education, 4(8), 63-71. http://www.eajournals.org/wp-content/uploads/Body-Image-andSelf-Esteem-Among-Isra'-University-Students.pdf

Reddy, S. L. (2013). Understanding the body image experiences of order men. International Journal of Humanities and Social Science, 3(9), 34-42. http://www.ijhssnet.com/journals/Vol_3_No_9_May_2013/4.pdf

Robins, R., Trzesniewski, K., Tracy, J., Gosling, S., \& Potter, J. (2002). Global selfesteem across the life span. Psychology and Aging, 17 (2), 423-434. https://doi.org/10.1037/0882$\underline{7974.17 .3 .423}$

Rodrigues, D., Teves, C., \& Medeiros, T. (2015). Autoimagem e satisfação corporal na adolescência. In T. Medeiros (Coord.). Adolescência: Desafios e riscos ( $2^{\mathrm{a}}$ ed. pp. 183-208). Letras Lavadas.

Romano, A., Negreiros, J., \& Martins, T. (2007). Contributos para a validação da escala de autoestima de Rosenberg numa amostra de adolescentes da região interior do país [Validation study of the Rosenberg self-esteem scale with adolescents of the north of Portugal]. Psicologia, $\quad$ Saúde $\quad$ e Doenças, $\quad 8(1), \quad$ 109-116. http://www.scielo.mec.pt/pdf/psd/v8n1/v8n1a08.pdf

Rosenberg, M. (1965). Society and the adolescent self-image. Princeton Legacy Library.

SantaBarbara, N. J., Whitworth, J. W., \& Ciccolo, J. T. (2017). A systematic review of the effects of resistance training on body image. Journal of Strength and Conditioning Research, 31(10), 2880-2888. https://doi.org/10.1519/JSC.0000000000002135

Santos, P. J., \& Maia, J. (2003). Análise factorial confirmatória e validação preliminar de uma versão portuguesa da escala de auto-estima de Rosenberg. Psicologia: Teoria, Investigação e Prática, $\quad$ 8(2), 253-268. https://repositorioaberto.up.pt/bitstream/10216/16170/2/SantoseMaia2003000077930.pdf

Simões, A. (1992). Ulterior validação de uma escala de satisfação com a vida (SWLS) [Ulterior validation of a life satisfaction scale]. Revista Portuguesa de Pedagogia, 26(3), 503-515. https://www.scienceopen.com/document?vid=5e0908d8-9da3-4ecb-ae05-ce077b5720f8

Souza, A. C., \& Alvarenga, M. S. (2016). Insatisfação com a imagem corporal em estudantes universitários - Uma revisão integrativa [Body dissatisfaction among university students An integrative review]. Jornal Brasileiro de Psiquiatria, 65(3), 286-299. https://doi.org/10.1590/0047-2085000000134 
Souza, L. R. M., Santos, C. A. V., Lima, S. F., Vasconcelos, A. C., \& Melo, G. F. (2013). O corpo na perspectiva masculina: a satisfação com a imagem corporal e sua relação com discrepância na percepção, IMC, escolaridade e idade [The body of the male perspective: satisfaction with body image and its relationship with discrepancy in perception, BMI, schooling and age]. Revista Brasileira de Ciência e Movimento, 21(2), 49-56. https://doi.org/10.18511/0103-1716/rbcm.v21n2p49-56

Talbot, D., Smith, E., \& Cass, J. (2019). The relationship between psychophysical body categorization performance and male body dissatisfaction. Scientific Reports, 9(1), 1-12. https://doi.org/10.1038/s41598-019-40502-z

Tylka, T. L., Bergeron, D., \& Schwartz, J. P. (2005). Development and psychometric evaluation of the Male Body Attitudes Scale (MBAS). Body Image, 2(2), 161-175. https://doi.org/10.1016/j.bodyim.2005.03.001

Tylka, T. L., \& Homan, K. J. (2015). Exercise motives and positive body image in physically active college women and men: Exploring an expanded acceptance model of intuitive eating. Body Image, 15, 90-97. https://doi.org/10.1016/j.bodyim.2015.07.003

Vargas, L. M., Herrera Cantorani, J. R., Mauricio, L. K., \& Annunciato, S. K. L. (2016). Nível de atividade física de estudantes universitários com e sem distúrbio da imagem corporal. Pensar a Prática, 19(1), 170-181. https://doi.org/10.5216/rpp.v19i1.35220

World Health Organization (Ed.). (2000). Obesity: Preventing and managing the global epidemic: report of a WHO consultation. World Health Organization. 